\title{
A hybridcontent based image retrieval system based onlocal binary pattern (LBP), color moment (CM) and edges
}

\author{
Hardeep Singh $^{\# 1}$, DheerajAgrawal ${ }^{* 2}$ \\ ${ }^{*}$ Research Scholar, Electronics and Communication Engineering, \\ Career Point University, Kota, Rajasthan, India \\ ${ }^{1}$ hardeep_singh1689@yahoo.co.in \\ *Assistant Professor, Electronics and Communication Engineering, \\ Maulana Azad National Institute of Technology, Bhopal, Madhya Pradesh, India
}

\begin{abstract}
In this paper a hybrid content based image retrieval system (CBIR) based on local binary pattern (LBP), color moment (CM) and edges have been efficiently used for image retrieval. LBP is used as it is sensible to noise so the neighboring pixels comparison is easy. CM is used as it is efficient in indexing the images based on the color in image retrieval system. Edges can be used to efficiently discover the shape information. So it will be better to combine all the three features and utilized the variable property of all the three. Wang database have been used for the experimentation. All the 10 classes are used for the results comparison. The results suggested that the proposed hybrid method have the capability over individual methods and also over the LBP and CM method in combination in efficient image retrieval.
\end{abstract}

Keyword-CBIR, Content Retrieval, LBP, CM, Edges

\section{INTRODUCTION}

Visual analysis is an emerging trend in research arena. CBIR system is more trending now a days because of the demand of efficient image retrieval system [1]. The demand of efficient image retrieval system is the need of the current edge so that picture information is extracted properly [2-4]. Image information solidifies the noisy pictures and data secluded from pictures through electronic or recreated estimations [5].

CBIR systems are similarly working with the same lay [6-10]. A section vector is ousted from each image in the database and the course of action of all highlight vectors is encircled as a database record [11-13]. At the time, a substitution vector is removed from the solicitation picture other than; it is energized against the section vectors in the record [14-16]. The key differentiation between the specific structures lies in the parts that they empty and in the counts that are utilized to take a gander at highlight vectors [17].

The shading is the most by and large used visual fragments as a piece of picture recuperation in light of the way that they are less requesting to withdraw isolated and surface and shape information. Shading highlight is acceptably ready to establishment multifaceted nature and free of picture size and presentation. Quantifiably, it demonstrates the joint probability of the intensities of the three shading channels [18]. Blends of timetables have been made for measuring strategy equivalence [19]. Most systems rely on upon in the wake of taking a gander at estimations of what are known as second-demand estimations enrolled from sales and set away pictures [20]. These figure measures of picture surface, for instance, the level of refinement, coarseness, directionality and consistency [21]; or periodicity, directionality and intercession [22]. Elective system for surface examination for picture recuperation merges the utilization of Gabor channels [23] and fractals [24].

The rest of the paper is organized in four different parts. Related work shows the latest literature in the related area. Method section explores the method used along with the algorithm. Result section discussed the results achieved along with the comparison. Conclusion section includes the concluding remarks based on the results achieved. Reference section is included at the end.

\section{RELATED WORKS}

In 2011, Jain et al. [25] suggested that the CBIR is a widely used procedure which is capable in finding images from huge image databases. With expansive information sets, there is probability of high dimensionality. In 2012, Das et al. [26] implemented a CBIR system which uses different feature of images by applying four methods, two methods were based on the color feature analysis and rest two were based on color and texture feature analysis using wavelet coefficients of an image. In 2007, Jain et al. [27] proposed an algorithm for retrieving images. The algorithm uses the shape information in an image along with its $3 \mathrm{D}$ information. The calculation utilizes the shape data as a part of a picture alongside its 3D data. In 2014, Wan et al. [28] suggested that the learning representations and similarity measures are very important for CBIR system. They have suggested that semantic gap issue which can be exists in the low-level image pixels which are only captured by 
machines and high-level semantic concepts perceived by human. In 2006, Heller et al. [29] presented a Bayesian framework for CBIR which is capable in modeling the distribution of color and texture features within sets of related images. They suggested that for a user specified content question the framework first concentrates an arrangement of images, from a named corpus, relating to that question. In 2012, Chaudhari et al. [30] suggested that the CBIR uses the visual properties of an image such as color, shape, texture, and spatial layout to represent and index the image. In 2015 Dubey et al. [31-34] suggested that data mining and evolutionary algorithms can be useful in improving the classification accuracy. So there used techniques may be helpful in image retrieval and proper classification. In 2007, Liu et al. [3] provided a comprehensive survey of the recent technical achievements in high-level semantic-based image retrieval. In 2015, Alzu'bi et al. [35] suggested that the multimedia contents complexity is increasing dominantly in the current digital world. So this generates the need of highly effective retrieval systems. In 2012, Jain et al. [36] presented an efficient framework with the combination of color, texture and shape information. They have applied the coordination of the above and bunch in view of alike properties. In view of five predominant colors they recover the comparative pictures. In 2013, Bhagat et al. [37] suggested that the execution of an electronic extensible design that is effortlessly indispensable with applications written in various dialects and linkable with various information sources. In [38] author has presented a brief survey on CBIR techniques.

\section{METHODS}

In this paper a hybrid approach based on LBP, CM and edge detection method are applied for the efficient image retrieval technique. LBP is used as it is efficient in thresholding the neighborhood for every pixel. By this classification accuracy can be improved and it can help in feature selection [39]. CM is useful in indexing the images based on the color in image retrieval system as it provides image retrieval based on color index. For result validation Wang database have been used [40] [41]. It is a collection of 1000 database.

In LBP method of image retrieval, the color images are converted to gray scale. As it works only on gray scale images. As we have already discussed that it is efficient in neighborhood pixel selection and thresholding. Then LBP values for the corresponding pixels are calculated. The corresponding pixel location is updated in the data matrix or used as the LBP mask vector. The neighboring pixel values are then compared with the central pixel value to calculate the pixel LBP value. It can be calculated for all the pixels. This can be done by traversing in the same order of clock or anti-clock wise position with the same position. So the comparison is depends on the pixels. It is started from the initial pixel. These values are between 0 to 255 . The main benefit of using LBP is translation invariant property.

CM is a supportive measurement which can be used to differentiate images based on the color feature. It is calculated based on the similarity of images. This can be basically determined by the normal distribution or by calculating their mean and variance. It shows a pattern of moment which can help in identifying the image based on color. Three moments are used mainly mean, standard deviation and skewness. The color can be defined as the hue, saturation and brightness. Then the moments are calculated for the separate channel in the picture

In the images unexpectedly changes are identified as the edges. It represents the local changes of the intensity. The benefit of the edge detection is that maximum of the shape information can be extracted from the edges as it encloses in the edges. Corners, lines and curves are the important features retrieved can be retrieved by the images. The intensity changes can be due to the changes in the surface, color, texture, orientation and may be because of inter reflections.

The following four steps are followed for the edge detection:

1. Smoothing reduces noise without restructuring the origin of edges.

2. Enhancement is applied for the image sharpening.

3. Detection by thresholding and edge histogram description.

4. Localization is used to detect the edges pixel location by edge.

The finite differences are calculated as follows:

$$
\begin{aligned}
& \mathrm{f}^{\prime}(\mathrm{x})=\lim _{h \rightarrow 0} \frac{f(\mathrm{x}+\mathrm{h})-\mathrm{f}(\mathrm{x})}{h} \\
& \mathrm{f}^{\prime}(\mathrm{x})=\mathrm{f}(\mathrm{x}+\mathrm{h})-\mathrm{f}(\mathrm{x}) \quad \text { for } \mathrm{h}=1 \\
& \text { Mask: [-1 1] }
\end{aligned}
$$

The masking of the edge model is [-1 01 1]. The second derivative can be calculated as follows:

$$
\begin{aligned}
& \mathrm{f}, '(\mathrm{x})=\lim _{h \rightarrow 0} \frac{f(\mathrm{x}+\mathrm{h})-\mathrm{f} /(\mathrm{x})}{h} \\
& \mathrm{f}^{\prime \prime}(\mathrm{x})=\mathrm{f}^{\prime}(\mathrm{x}+1)-\mathrm{f}^{\prime}(\mathrm{x}) \\
& \mathrm{f})^{\prime \prime}(\mathrm{x})=\mathrm{f}(\mathrm{x}+2)-2 \mathrm{f}(\mathrm{x}+1)+\mathrm{f}(\mathrm{x}) \\
& \text { for } \mathrm{h}=1
\end{aligned}
$$

It is evaluated center by placing $x$ in the place of $x+1$. 
$\mathrm{f}^{\prime \prime}(\mathrm{x})=\mathrm{f}(\mathrm{x}+1)-2 \mathrm{f}(\mathrm{x})+\mathrm{f}(\mathrm{x}-1)$

The masking for the edge model is [1 -21$]$. The edge detection is then calculated based on the magnitude and direction by the following:

$$
\begin{gathered}
\nabla f=\left(\begin{array}{c}
\frac{\partial f}{\partial x} \\
\frac{\partial f}{\partial y}
\end{array}\right) \\
\mathrm{M}(\nabla f)=\sqrt{\left(\frac{\partial y}{\partial x}\right)^{2}+\left(\frac{\partial y}{\partial x}\right)^{2}} \\
\mathrm{M}(\nabla f)=\sqrt{M_{x}^{2}+M_{y}^{2}} \\
\mathrm{~d}(\nabla f)=\tan ^{-1} \frac{M_{y}}{M_{x}}
\end{gathered}
$$

The approximated magnitude of gradient can be used as:

$\mathrm{M}(\nabla f)=\left|M_{x}\right|+\left|M_{y}\right|$

The strength of the edge can be determined by the magnitude. The following steps are needed for magnitude in the edge detection.

$$
\begin{gathered}
\hat{f}(\mathrm{x}, \mathrm{y})=f(\mathrm{x}, \mathrm{y}) * G(\mathrm{x}, \mathrm{y}) \\
\widehat{f_{x}}=\hat{f}(\mathrm{x}, \mathrm{y}) * M_{x}(\mathrm{x}, \mathrm{y}) \\
\widehat{f_{y}}=\hat{f}(\mathrm{x}, \mathrm{y}) * M_{y}(\mathrm{x}, \mathrm{y}) \\
\mathrm{M}(\mathrm{x}, \mathrm{y})=\left|\widehat{f_{x}}\right|+\left|\widehat{f_{y}}\right| \\
\mathrm{D}(\mathrm{x}, \mathrm{y})=\tan ^{-1} \frac{\widehat{f_{y}}}{\widehat{f_{x}}}
\end{gathered}
$$

\section{Proposed Algorithm:}

Input: Select the input image from the Wang database.

Output: Total number of similar query image retrieved.

Step 1: Image preprocessing is done in the first step. Here image query is denoted by $\mathrm{I}_{\mathrm{q}}$.

Step 2: Color features is then extracted base on the color moment method.

Suppose there are n observations like $X_{1}, X_{2}, \ldots, X_{n}$ belong to $X$ variable. The average of $X_{1}, X_{2}, \ldots, X_{n}$ can be represented as is the mean, $\mu_{X}$, denoted as follows:

$$
\mu_{X}=\bar{X}=\frac{1}{n} \sum_{i=1}^{n} X_{i}
$$

Then the standard deviation can be calculated as follows:

$$
\begin{aligned}
\sigma_{X} & =\sqrt{\sigma_{X}^{2}} \text {. We have } \\
\sigma_{X} & =\sqrt{\frac{1}{n}\left\{\sum_{i=1}^{n} X_{i}^{2}-\frac{1}{n}\left(\sum_{i=1}^{n} X_{i}\right)^{2}\right\}}
\end{aligned}
$$

According to the Karl,skewness can be defined as follows:

$$
s k=\frac{\mu-\text { mode }}{\sigma}
$$

$\mathrm{A}=\mathrm{CM}\left(\mathrm{I}_{q}\right)$

Step 3: For extracting the texture feature LBP method have been applied.

$\operatorname{LBP}\left(\mathrm{X}_{\mathrm{c}}, \mathrm{Yc}\right)=\sum_{n=0}^{n-1} 2^{n} \mathrm{Q}\left(\mathrm{I}_{\mathrm{n}}-\mathrm{I}_{\mathrm{c}}\right)$

Where $\mathrm{X}_{\mathrm{c}}$ and $\mathrm{Y}_{\mathrm{c}}$ denotes the central pixel. Q can be understood as follows:

$$
\mathrm{Q}(\mathrm{X})=\left\{\begin{array}{rr}
1 & \text { if } x \geq 0 \\
0 & \text { else }
\end{array}\right.
$$

$I_{n}$ denote the neighbor pixel intensity. $I_{c}$ denotes the central pixel intensity. 
For the calculated center vector $\mathrm{X}_{\mathrm{c}}$ and $\mathrm{Y}_{\mathrm{c}}$ the position of the neighbor in the LBP can be calculated as follows:

$$
\begin{aligned}
& \mathrm{X}_{\mathrm{n}}=\mathrm{X}_{\mathrm{c}}+\mathrm{R} \cos \frac{2 \pi n}{s} \\
& \mathrm{Y}_{\mathrm{n}}=\mathrm{Y}_{\mathrm{c}}-\mathrm{R} \sin \frac{2 \pi n}{S}
\end{aligned}
$$

Where $\mathrm{R}$ denotes radius and $\mathrm{S}$ is the sample point.

$$
\mathrm{B}=\operatorname{LBP}\left(\mathrm{I}_{\mathrm{q}}\right)
$$

Step 4: Edge features have been extracted for the shape information.

$$
\mathrm{C}=\mathrm{E}\left(\mathrm{I}_{\mathrm{q}}\right)
$$

Step 5: Query image vector has been created with the color and texture feature.

$$
\mathrm{V}_{\mathrm{q}}=\{\mathrm{A}, \mathrm{B}, \mathrm{C}\}
$$

Step 6: step 2 to 5 are repeated for the entire query index.

Step 7: Perform similarity measure.

For similarity measurement from the experimental images can be calculated as follows by the Euclidean distance, d using equation below:

$$
d=\sqrt{\left(F_{Q}[i]-F_{D B}[i]\right)^{2}}
$$

$F_{Q}[i]$ denotes $i^{\text {th }}$ feature of the query image and the $F_{D B}[i]$ indicates the related feature in the feature vector.

Step 8: Determine the similarity by the given threshold values.

Step 9: Store the results.

The group of color extracted based on the dominant color which is the combination of color fetched. Before separating the color attributes of a picture, all pixels on database pictures are arranged into comparable sorts of gatherings as per the similarity of their hues. A color will be chosen from predefined hues which are exceptionally close to picture pixel color and it is stored as a new pixel. Color distance $\mathrm{C}_{\mathrm{D}} \mathrm{can}$ be measured by adopting Euclidean distance equation as specified below:

$$
C_{D}=\min \left(\sqrt{\left(R_{P}-R_{i T}\right)^{2}+\left(G_{P}-G_{i T}\right)+\left(B_{P}-B_{i T}\right)^{2}}\right)
$$

$R_{P}, G_{P}$ and $B_{P}$ are the color intensity values of red, green, and blue colors respectively. $R_{i T}, G_{i T}$ andB $B_{i T}$ are the color table index. The maximum percentage color component is selected as the dominant color and stored.

For similarity measurement from the experimental images can be calculated as follows by the Euclidean distance, d using equation below:

$$
d=\sqrt{\left(F_{Q}[i]-F_{D B}[i]\right)^{2}}
$$

$F_{Q}[i]$ denotes $i^{\text {th }}$ feature of the query image and the $\mathrm{F}_{\mathrm{DB}}$ [i] indicates the related feature in the feature vector. Fig. 1 shows the flowchart and working mechanism of our proposed work. It clearly indicate the process of feature extraction, indexed feature vector calculation then the process of similarity measurement is performed and finally similar images are retrieved.

It can be said that in color moment the images are first selected and moment for the query images are calculated then dominant color is used for the color feature extraction and then similarity is calculated based on the rank. In this paper we have combined the above methodologies for achieving the better results.

\section{RESULTS AND DISCUSSION}

The results are compared based on the Wang database. All the ten classes are considered for experimentation. Fig. 2 and fig. 3 shows the result obtained after applying LBP and CM method. Different variations have been found in the results. It can suggest that the results are varying based on the properties of the images. Fig. 4 shows the result obtained after applying LBP and CM method combined. Fig. 5 shows the result based on the proposed approach which is the combination LBP, CM and edge detection. Fig. 6, fig. 7 and fig.8 show the results of $\mathrm{LBP}, \mathrm{CM}$ and $\mathrm{LBP}+\mathrm{CM}$ respectively for the selected images from the database. The overall results are shown in fig. 9. In case of LBP, CM and LBP + CM the results obtained are varying. Means the results are different for different images and good results are obtained case by case. But in case of the proposed method which is the combination of LBP, CM and edges method better results have been found in an average cases considered. 


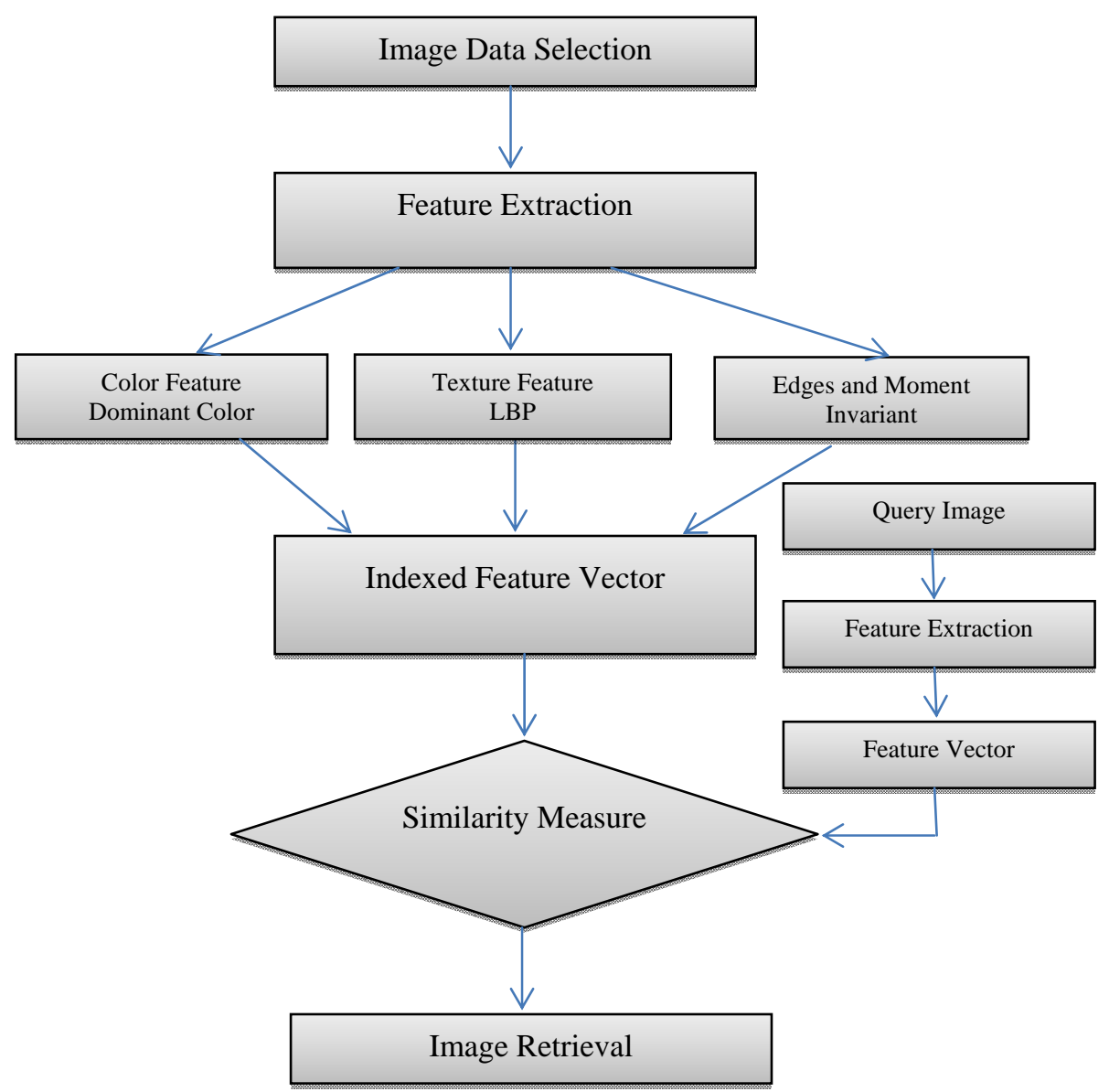

Fig.1. Working flowchart

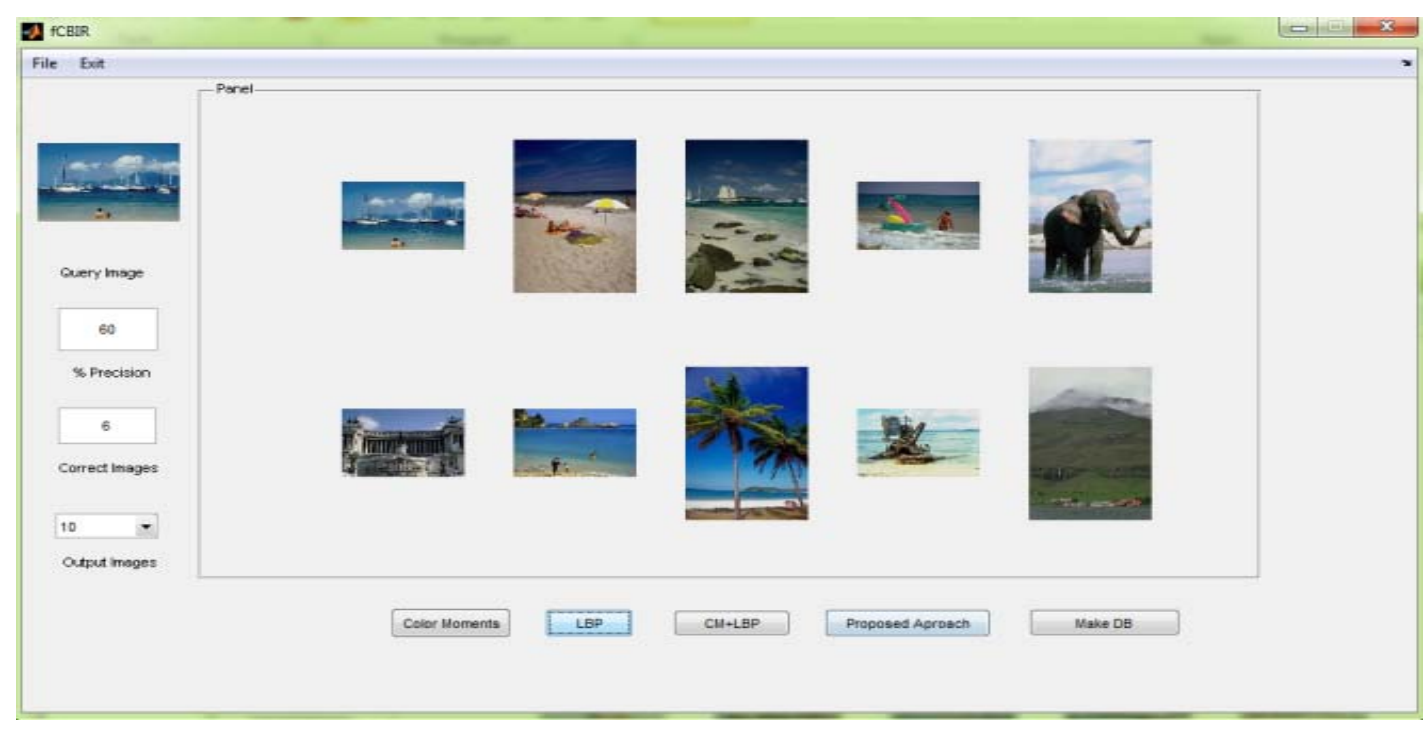

Fig.2. LBP precision for sea image database 


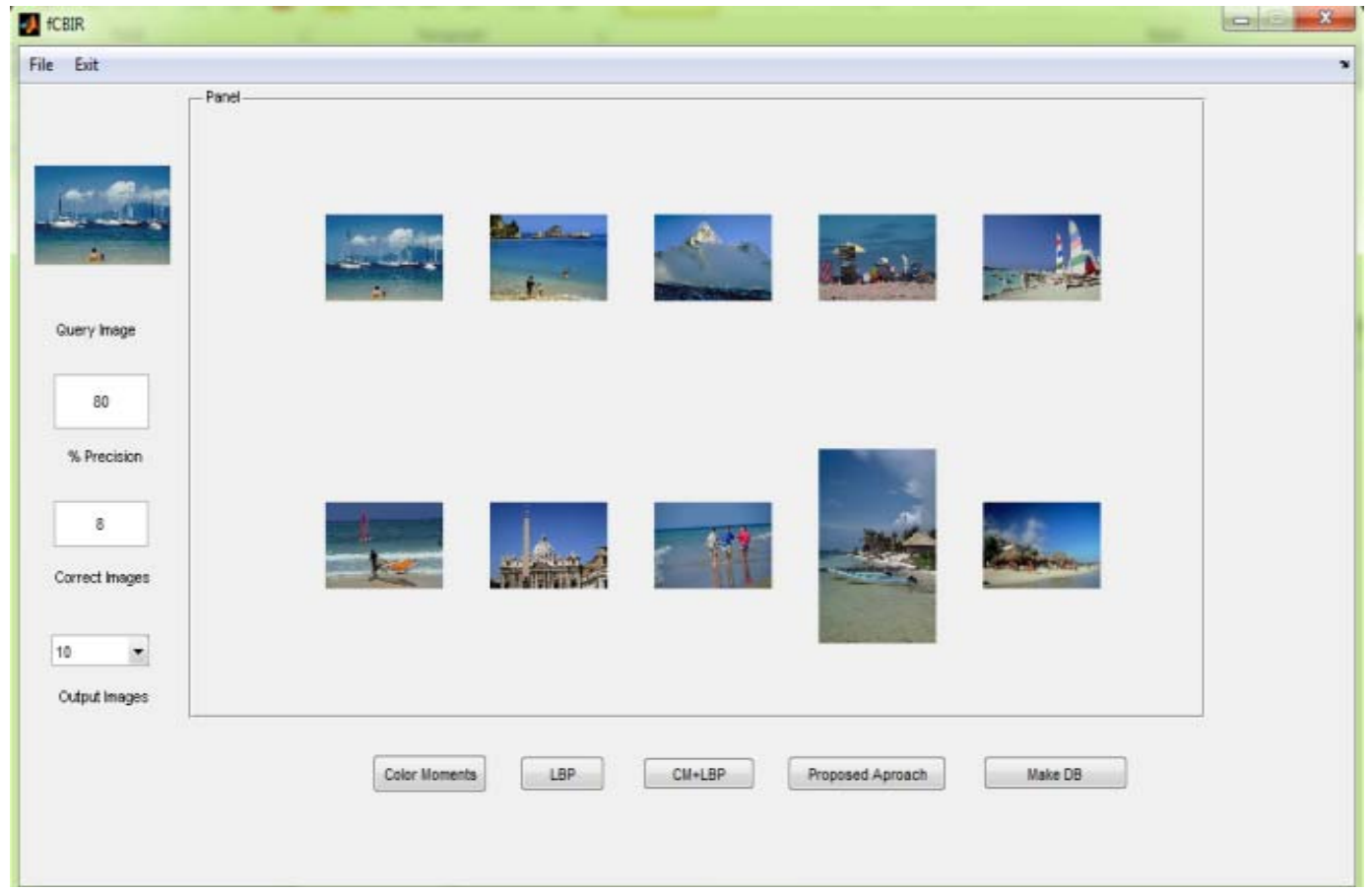

Fig.3. CM for image database

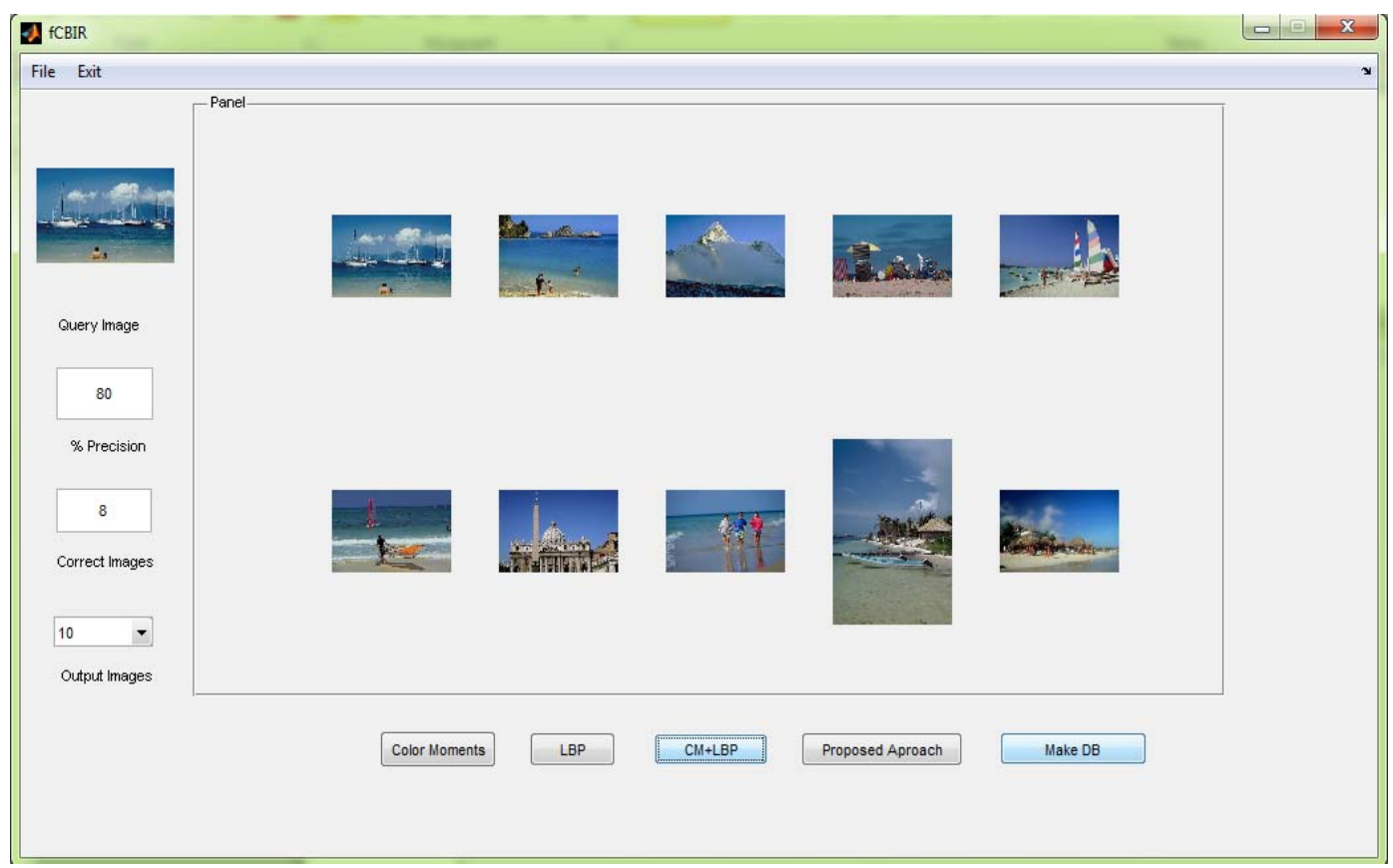

Fig.4. LBP + CM for image database 


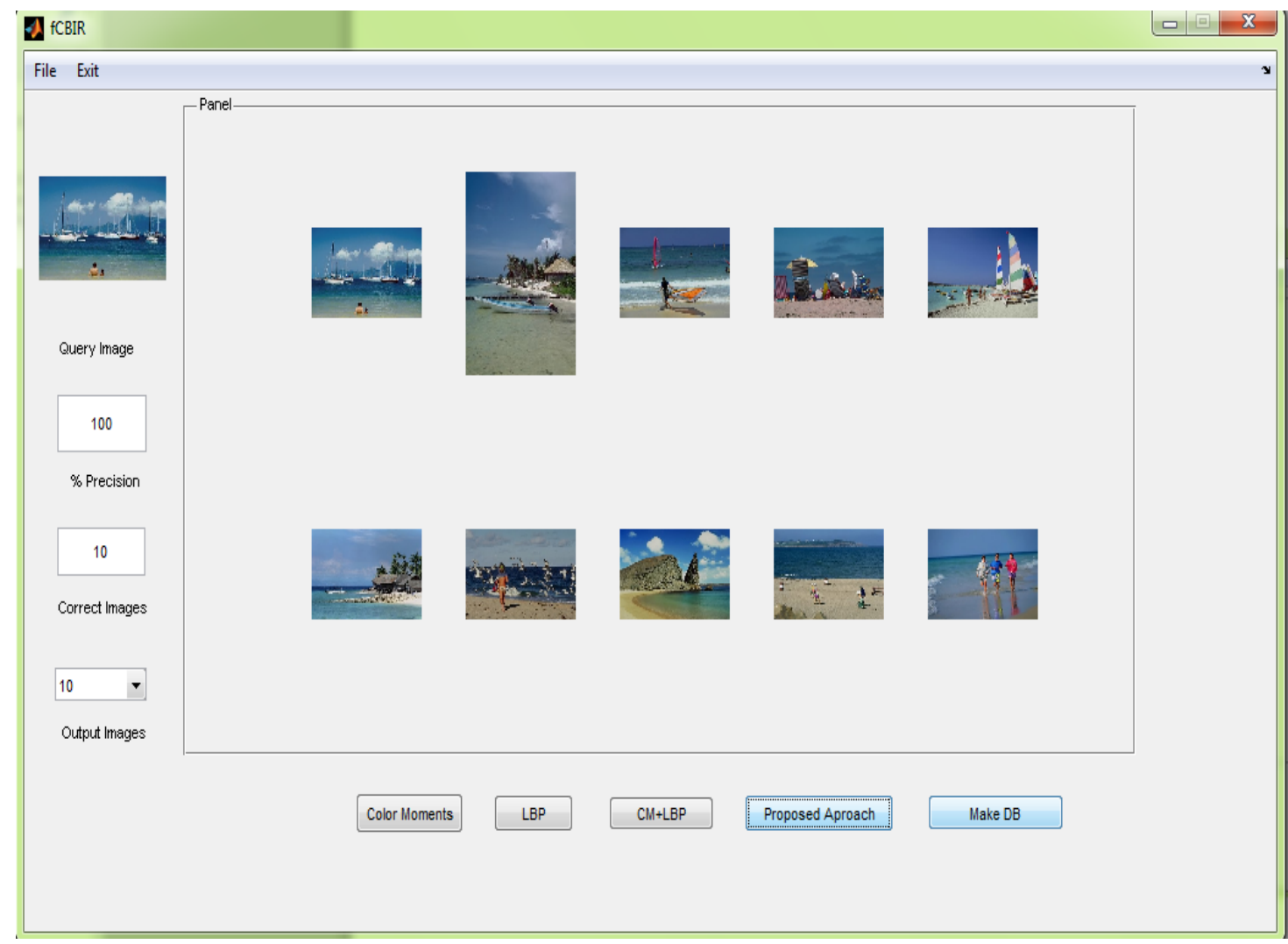

Fig.5. Proposed method for the image database

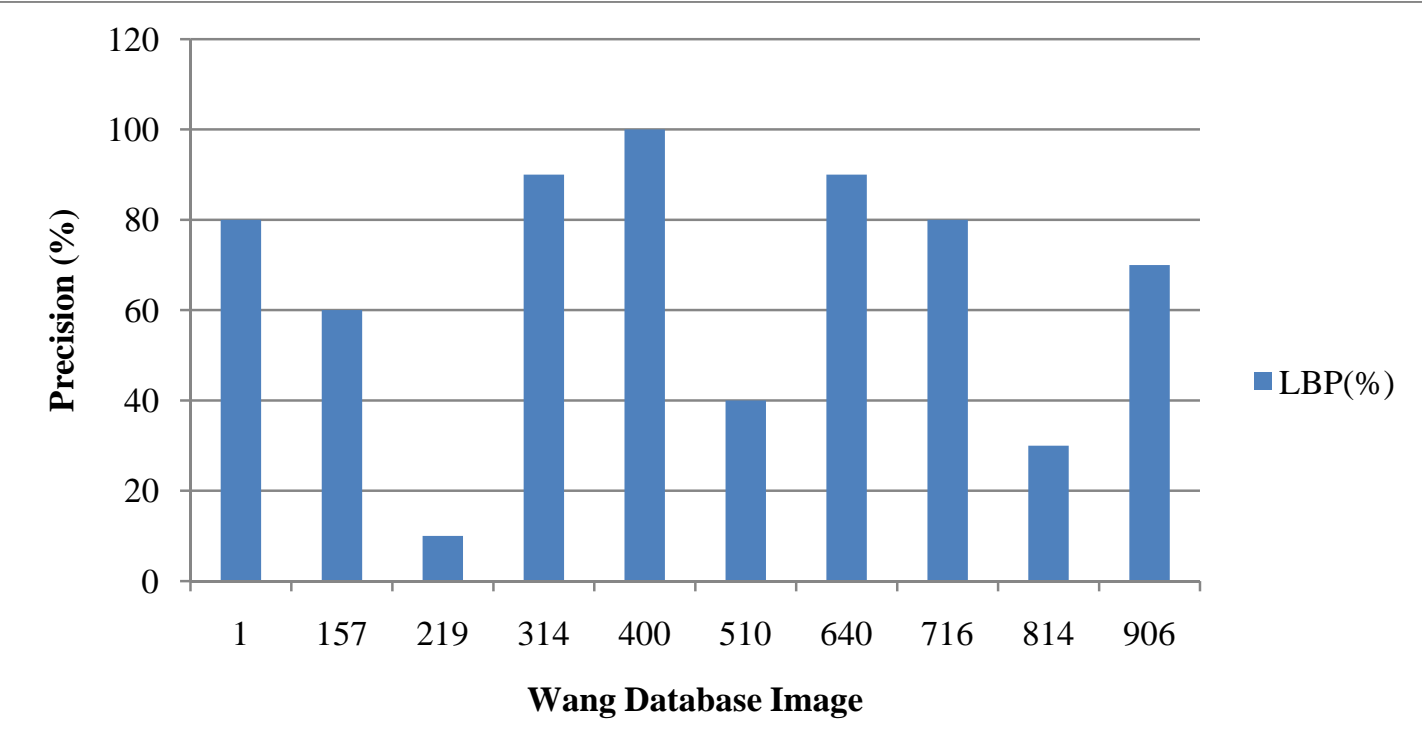

Fig.6. Precision results of LBP method on the selected images from Wang database 


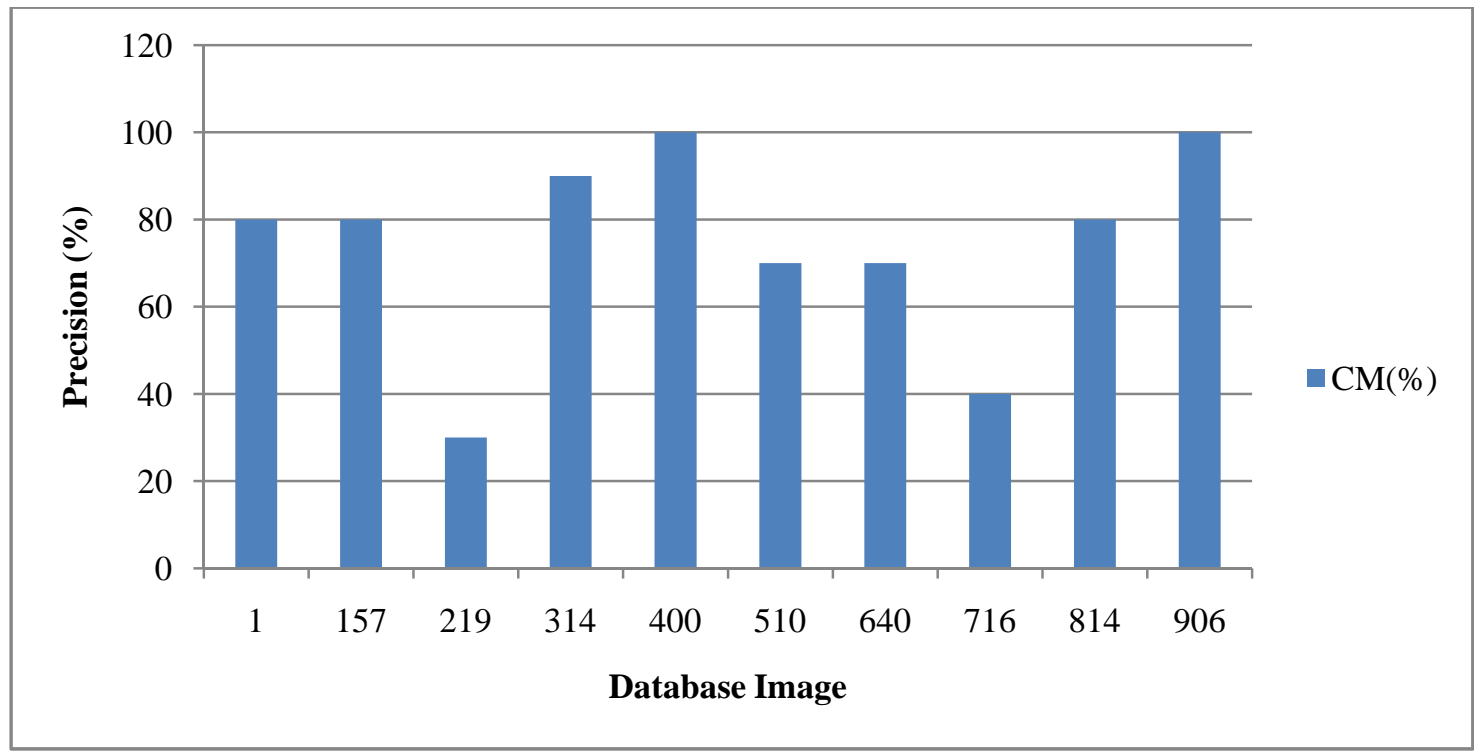

Fig.7. Precision results of CM method on the selected images from Wang database

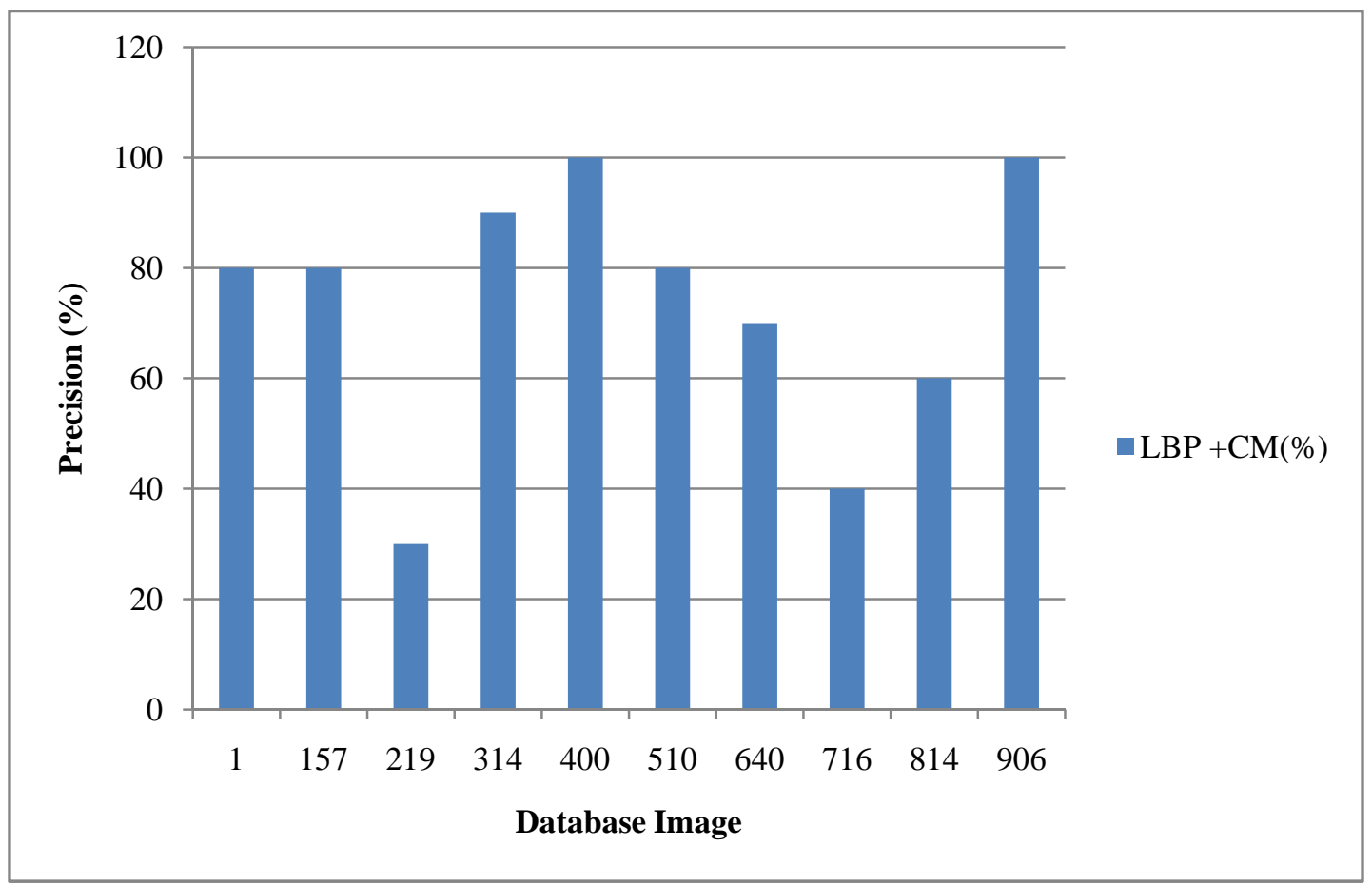

Fig.8. Precision results of LBP + CM method on the selected images from Wang database 


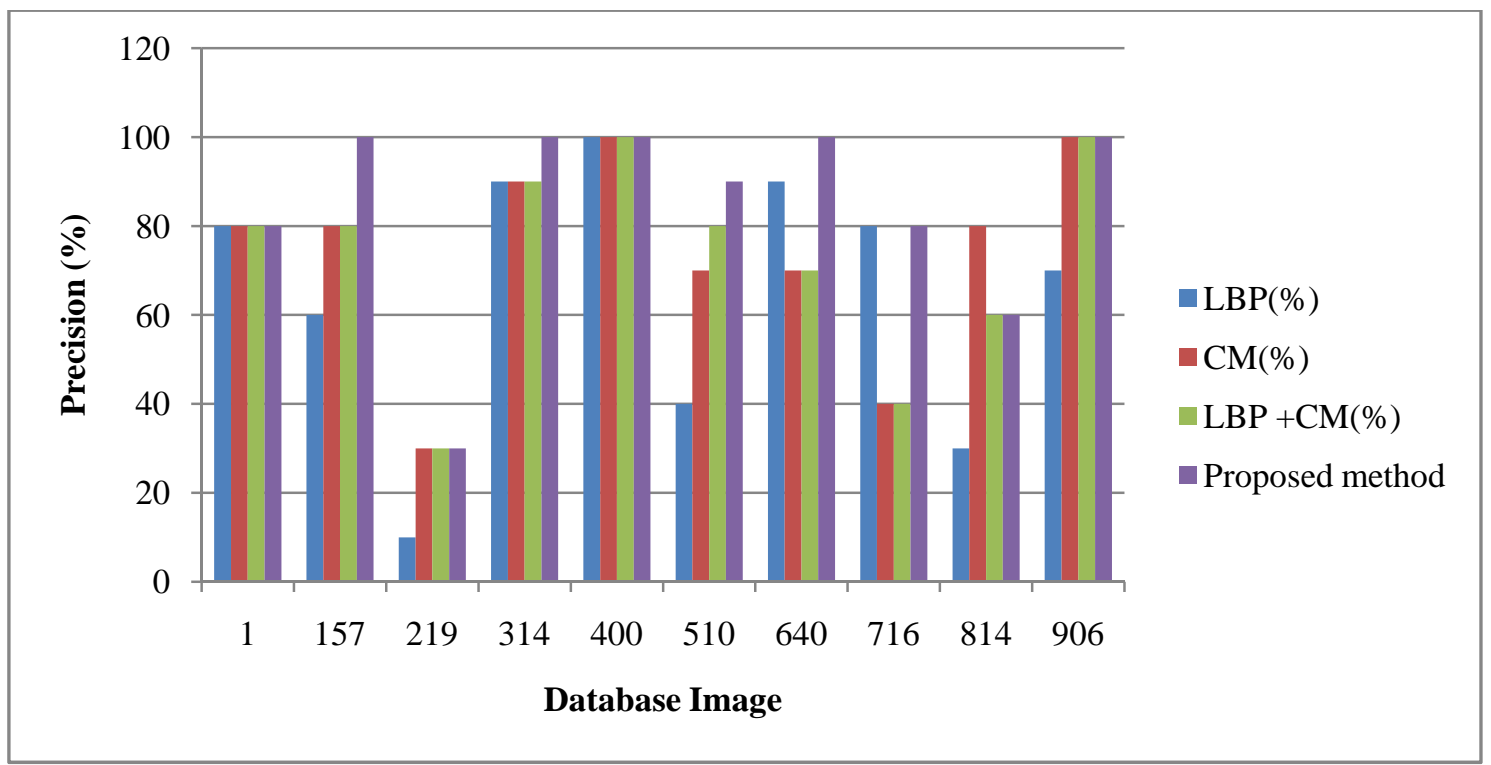

Fig.9. Precision results comparison on the selected images from Wang database

\section{CONCLUSIONS}

In this paper LBP, CM, LBP + CM and the combination of LBP, CM and edges for the image retrieval based on CBIR system have been analyzed and discussed. The results have been discussed based on the individual capability of LBP, CM and edges along with the combination altogether. The individual results are different in different cases and in some cases LBP and in some case CM have shown better results. But in the case of hybridization of the three methods better results have been obtained in comparison to using it individually.

\section{REFERENCES}

[1] Fu YQ, Wang YS. An algorithm for edge detection of gray-scale image based on mathematical morphology [J]. Journal of Harbin Engineering University. 2005; 5:027.

[2] Buades A, Coll B, Morel JM. A review of image denoising algorithms, with a new one. Multiscale Modeling \& Simulation. 2005; 4(2):490-530.

[3] Liu Y, Zhang D, Lu G, Ma WY. A survey of content-based image retrieval with high-level semantics. Pattern recognition. 2007 Jan 31; 40(1):262-82.

[4] Prasanna MK, Rai SC. Image Processing Algorithms-A Comprehensive Study. International Journal of Advanced Computer Research. 2014 Jun 1; 4(2):532.

[5] Anandan P, Sabeenian RS. Curvelet based Image Compression using Support Vector Machine and Core Vector Machine-A Review. International Journal of Advanced Computer Research. 2014 Jun 1; 4(2):673.

[6] Kato T. Database architecture for content-based image retrieval. InSPIE/IS\&T 1992 symposium on electronic imaging: science and technology 1992 Apr 1 (pp. 112-123). International Society for Optics and Photonics.

[7] Flickner M, Sawhney H, Niblack W, Ashley J, Huang Q, Dom B, Gorkani M, Hafner J, Lee D, Petkovic D, Steele D. Query by image and video content: The QBIC system. Computer. 1995 Sep; 28(9):23-32.

[8] Corea F. Emotional speculative behavior in the option market. International Journal of Advanced Computer Research. 2016 Jan 1; 6(22):18.

[9] Pentland A, Picard RW, Sclaroff S. Photobook: Content-based manipulation of image databases. International journal of computer vision. 1996 Jun 1; 18(3):233-54.

[10] Smith JR, Chang SF. VisualSEEk: a fully automated content-based image query system. In Proceedings of the fourth ACM international conference on Multimedia 1997 Feb 1 (pp. 87-98). ACM.

[11] Wang JZ, Wiederhold G, Firschein O, Wei SX. Content-based image indexing and searching using Daubechies' wavelets. International Journal on Digital Libraries. 1998 Mar 1;1(4):311-28.

[12] Carson C, Belongie S, Greenspan H, Malik J. Blobworld: Image segmentation using expectation-maximization and its application to image querying. IEEE Transactions on Pattern Analysis and Machine Intelligence. 2002 Aug; 24(8):1026-38.

[13] Wang JZ, Li J, Wiederhold G. SIMPLIcity: Semantics-sensitive integrated matching for picture libraries. IEEE Transactions on pattern analysis and machine intelligence. 2001 Sep; 23(9):947-63.

[14] Puviarasan N, Bhavani R. Retrieval of Images Using Weighted Features. International Journal of Advanced Computer Research. 2014 Mar 1; 4(1):60.

[15] Patidar D, Jain N, Nagariya B, Mishra M. Image Classification by Combining Wavelet Transform and Neural Network. International Journal of Advanced Computer Research. 2013 Dec 1; 3(4):106.

[16] Singh H, Kaur T. Novel Method for Edge Detection for Gray Scale Images using VC++ Environment. International Journal of Advanced Computer Research. 2013 Dec 1; 3(4):193.

[17] Mathur A, Mathur R. Content Based Image Retrieval by Multi Features using Image Blocks. International Journal of Advanced Computer Research. 2013 Dec 1; 3(4):251.

[18] Viswa SS. Efficient retrieval of images for search engine by visual similarity and re ranking. International Journal of Advanced Computer Research. 2013 Jun 1; 3(2):47.

[19] Eakins JP, Graham ME. Content based image retrieval: A report to the JISC technology applications programme. Cyberabstracts.

[20] Tamura H, Mori S, Yamawaki T. Textural features corresponding to visual perception. IEEE Transactions on Systems, Man, and Cybernetics. 1978 Jun; 8(6):460-73. 
[21] Zhang S, Bao CW, Shen HB. Independent component analysis based on adaptive artificial bee colony. International Journal of Advanced Computer Research. 2016; 6(25):146-152.

[22] Liu F, Picard RW. Periodicity, directionality, and randomness: Wold features for image modeling and retrieval. IEEE transactions on pattern analysis and machine intelligence. $1996 \mathrm{Jul}$; 18(7):722-33.

[23] Kaplan LM, Murenzi R, Namuduri KR. Fast texture database retrieval using extended fractal features. InPhotonics West'98 Electronic Imaging 1997 Dec 23 (pp. 162-173). International Society for Optics and Photonics.

[24] Smith JR. Integrated spatial and feature image system: Retrieval, analysis and compression [Ph D dissertation]. Columbia University, New York. 1997.

[25] Jain M, Singh SK. A survey on: content based image retrieval systems using clustering techniques for large data sets. International Journal of Managing Information Technology. 2011 Nov 1;3(4):23.

[26] Das S, Garg S, Sahoo G. Comparison of Content Based Image Retrieval Systems Using Wavelet and Curvelet Transform. The International Journal of Multimedia \& Its Applications. 2012 Aug 1; 4(4):137.

[27] Jain A, Muthuganapathy R, Ramani K. Content-based image retrieval using shape and depth from an engineering database. In International Symposium on Visual Computing 2007 Nov 26 (pp. 255-264). Springer Berlin Heidelberg.

[28] Wan J, Wang D, Hoi SC, Wu P, Zhu J, Zhang Y, Li J. Deep learning for content-based image retrieval: A comprehensive study. In Proceedings of the 22nd ACM international conference on Multimedia 2014 Nov 3 (pp. 157-166). ACM.

[29] Heller KA, Ghahramani Z. A simple Bayesian framework for content-based image retrieval. In2006 IEEE Computer Society Conference on Computer Vision and Pattern Recognition (CVPR'06) 2006 (Vol. 2, pp. 2110-2117). IEEE.

[30] Chaudhari R, PatilAM. Content based image retrieval using color and shape features. International Journal of Advanced Research in Electrical, Electronics and Instrumentation Engineering. 2012 Nov; 1(5).

[31] Dubey AK, Gupta U, Jain S. Breast cancer statistics and prediction methodology: a systematic review and analysis. Asian Pacific journal of cancer prevention: APJCP. 2014 Dec; 16(10):4237-45.

[32] Dubey AK, Gupta U, Jain S. A Survey on Breast Cancer Scenario and Prediction Strategy. InProceedings of the 3rd International Conference on Frontiers of Intelligent Computing: Theory and Applications (FICTA) 20142015 (pp. 367-375). Springer International Publishing.

[33] Dubey AK, Gupta U, Jain S. Epidemiology of lung cancer and approaches for its prediction: a systematic review and analysis. Chinese Journal of Cancer. 2016 Jul 30; 35(1):1.

[34] Dubey AK, Gupta U, Jain S. Analysis of k-means clustering approach on the breast cancer Wisconsin dataset. International Journal of Computer Assisted Radiology and Surgery. 2016 Jun 16:1-5.

[35] Alzu'bi A, Amira A, Ramzan N. Semantic content-based image retrieval: A comprehensive study. Journal of Visual Communication and Image Representation. 2015 Oct 31; 32: 20-54.

[36] Jain N, Sharma S, Sairam RM. Result Analysis on Content Base Image Retrieval using Combination of Color, Shape and Texture Features. International Journal of Advanced Computer Research (IJACR). 2012;2.

[37] Bhagat AP, Atique M. Web based image retrieval system using color, texture and shape analysis: comparative analysis. International Journal of Advanced Computer Research. 2013 Sep 1; 3(3):58.

[38] Dubey A. Efficient Content based Image Retrieval (CBIR) Techniques: A Survey. ACCENTS Transactions on Image Processing and Computer Vision (TIPCV). 2015; 1(1):28-32.

[39] Kumar E, Khan Z, Jain A. A review of content based image classification using machine learning approach. International Journal of Advanced Computer Research. 2012; 2(5):55-60.

[40] Li J, Wang JZ. Automatic linguistic indexing of pictures by a statistical modeling approach. IEEE Transactions on pattern analysis and machine intelligence. 2003 Sep; 25(9):1075-88.

[41] Wang JZ, Li J, Wiederhold G. SIMPLIcity: Semantics-sensitive integrated matching for picture libraries. IEEE Transactions on pattern analysis and machine intelligence. 2001 Sep; 23(9):947-63. 